\title{
Características Espermáticas de Sêmen Resfriado de Suíno e Conservado em Diferentes Diluentes 1
}

\author{
Ana Maria Martins Alves Vasconcelos ${ }^{2}$, Gentil Vanini de Moraes $^{3}$, Ivan Moreira ${ }^{3}$, \\ Luís Paulo Rigolon ${ }^{3}$, Elias Nunes Martins ${ }^{4}$
}

\begin{abstract}
RESUMO - Utilizaram-se 36 ejaculados de três cachaços, diluídos em partes iguais, com os diluentes Beltsville Thawing Solution (BTS), Zorlesco-modificado (ZOR) e BTZOR (desenvolvido na Universidade Estadual de Maringá), para avaliar as características do sêmen e o período de conservação até $25 \%$ de motilidade progressiva (MP) para cada diluente. O modelo estatístico foi de blocos inteiramente casualizados, bloqueando cachaço/mês. O período de conservação foi maior no BTZOR (4,94 $\pm 0,30$ dias), seguido pelos ZOR (4,62 $\pm 0,29$ dias) e BTS (3,27 $\pm 0,30$ dias). Análises de regressão mostraram comportamento quadrático na MP para os meios BTS e ZOR e cúbico para o BTZOR, com o decorrer dos dias de conservação. O vigor (V) apresentou comportamento linear para BTS e ZOR e quadrático para BTZOR. O grau de aglutinação apresentou comportamento linear para todos os diluentes. O BTZOR conservou sêmen por maior período de tempo, mantendo melhores médias de motilidade progressiva e vigor, seguido pelos ZOR e BTS.
\end{abstract}

Palavras-chave: diluentes, período de conservação, sêmen, suíno

\section{Spermatic Characteristics of Cooled and Conservated Swine Semen in Different Diluents}

ABSTRACT - Thirty-six ejaculates from three boars, equally diluted in the diluents Beltsville Thawing Solution (BTS), Modified Zorlesco (ZOR) and BTZOR (developed at Universidade Estadual de Maringá), were used to evaluate the characteristics of each ejaculate and the semen conservation period until $25 \%$ of progressive motility (PM) for each diluents. The experimental design was completely randomized blocks, blocking boar/month. The better conservation period was BTZOR ( $4.94 \pm 0.30$ days), followed by ZOR (4.62 \pm 0.29 days) and BTS (3.27 \pm 0.30 days). Regression analyses showed a quadratic behavior for BTS and ZOR and cubic behavior for BTZOR to PM, as the days for conservation go through. Vigor (V) showed linear behavior to BTS and ZOR and quadratic behavior for BTZOR. Degree agglutination showed linear behavior for all diluents. The BTZOR maintained the quality for more days, maintaining the best averages of PM and V, followed by ZOR and BTS.

Key Words: diluents, conservation days, semen, swine

\section{Introdução}

O bom desenvolvimento da inseminação artificial (IA) de suínos, a preparação do diluente, a conservação e a utilização do sêmen diluído devem implicar em atividades simples e viáveis para o produtor, com tempo de conservação do sêmen suficiente para a execução da IA, devendo o diluente ser de baixo custo (BARITEAU et al., 1977; PAQUIGNON et al., 1987).

O êxito da IA está relacionado à capacidade do diluente em conservar os espermatozóides em condições adequadas durante a estocagem do sêmen (JOHNSON et al., 1988; WOELDERS, 1992). Nesse sentido, PAQUIGNON et al. (1987) destacaram a necessidade de realizar estudos efetivos em busca de diluentes que possam preservar as qualidades fecun- dantes do sêmen diluído de cachaços por maior período de tempo possível, a fim de otimizar o uso do sêmen, melhorar o manejo e causar menor desgaste aos reprodutores.

Os diluentes mais amplamente utilizados para diluição e conservação do sêmen sob resfriamento são Kiev (JOHNSON et al., 1982; SIL VEIRA, 1983), etileno diamino tetracetato (EDTA) e Zorlesco (PAQUIGNON et al., 1987) e Beltsville Thawing Solution - BTS (WOELDERS, 1992).

Várias formulações de diluentes foram testadas e usadas em sêmen de cachaço, considerando-se índice de prenhez e tamanho de leitegada. Contudo, foram encontrados resultados consistentes com o uso do BTS, o qual contém apenas número limitado de ingredientes, de baixo custo, parecendo ser o favo-

\footnotetext{
${ }^{1}$ Parte da dissertação de metrado da primeira autora apresentada à UEM.

2 Eng a Agrônoma, Mestre em Zootecnia. E.mail: amavasco@terra.com.br

3 Professor do DZO da Univesidade Estadual de Maringá (UEM). E.mail:gvmoraes@uem.br; imoreira@uem.br; rig@wnet.com.br

4 Professor do DZO da UEM e pesquisador do CNPq. E.mail: enmartins@uem.br
} 
rito na IA e na estocagem do sêmen diluído durante um número limitado de dias (WOELDERS, 1992).

WOELDERS (1992) citou ainda que a MP e a preservação do sêmen durante o transporte parecem ser parâmetros dependentes da composição do meio. Reportagens no PIG INTERNATIONAL (1994) mostraram que as taxas de diluições são ajustadas de acordo com as porcentagens de células normais e da MP.

PEDERSEN et al. (1995) recomendaram o uso de sêmen que apresente pelo menos $70 \%$ de MP inicial e não mais que $30 \%$ de anormalidades. ZAVOS et al. (1996) também mostraram que a qualidade do sêmen é, geralmente, avaliada pela contagem do número de espermatozóides e da MP.

WABERSKI et al. (1994) estudaram o potencial de fecundação do sêmen diluído e conservado em Androhep e BTS por até 120 horas e constataram MP de 92,6\% (0-24h), 90,0\% (24-48h), 82,4\% (48-72h), 77,9\% (72-96h), 73,0\% (96-120h) quando o sêmen foi diluído em meio Androhep e os resultados de conservação de sêmen com o BTS foram de 92,0; 87,$3 ; 77,1 ; 71,8$ e $61,7 \%$ de MP, respectivamente.

ALEXOPOULOS et al. (1996) utilizaram sêmen de três cachaços diluído em BTS e doses inseminantes que continham um, três e cinco bilhões de espermatozóides. Observaram que o índice de MP diminuiu significativamente a partir de 48 horas até 120 horas de conservação do sêmen, sendo que a queda mais acentuada ocorreu com doses inseminantes de cinco bilhões de espermatozóides.

Em um trabalho sobre avaliação de sêmen de cachaço, o qual foi diluído em BL-1 (semelhante ao BTS) e mantido a $17^{\circ} \mathrm{C}$ para análises, quanto à porcentagem de MP no sétimo dia, XU et al. (1996) obtiveram um percentual de MP inicial de três cachaços que variou de 88,85 a $81 \%$, respectivamente. Porém, no sétimo dia de conservação do sêmen, a redução no percentual de MP foi de 10,9; 14,3 e $35,8 \%$ para cada ejaculado dos respectivos cachaços, considerando que o último cachaço apresentou o menor volume de sêmen.

Diante das diferentes respostas quanto à conservação de sêmen resfriado de suíno, realizou-se esta pesquisa com a finalidade de avaliar o uso de três diluentes por meio das características de cada ejaculado e do período de conservação do sêmen de suíno com MP mínima de $25 \%$, preservado a $15^{\circ} \mathrm{C}$ e diluído em BTS, Zorlesco-modificado (ZOR) e BTZOR, meio desenvolvido na Universidade Estadual de Maringá (UEM).

\section{Material e Métodos}

O experimento foi conduzido na Fazenda Experimental de Iguatemi (FEI) e no Laboratório de Reprodução Animal da UEM, em Maringá-PR, de março de 1996 a fevereiro de 1997. Durante 12 meses, foram realizadas 12 colhetas por cachaço, sendo um da raça Large White, um Landrace e um Duroc, com idades entre 10 e 48 meses. Os animais foram mantidos em instalações convencionais para a criação de suínos, do tipo semi-confinada. Os reprodutores receberam em duas refeições, de 1,8 a 2,0 kg de ração de gestação por dia, contendo $13,55 \%$ de PB e 3,02 Mcal de energia metabolizável por quilo de ração, contendo núcleo mineral e vitamínico Suiplus (NUTRIS).

Um dia antes da coleta do sêmen, os cachaços foram submetidos a "toillet" completa, sendo banhados e aparados os pêlos do óstio prepucial. No momento da colheita, houve a higienização do prepúcio com uma solução de desinfetante (Kilol-L- Chimie Brasileira Ind. e Com. Ltda) e água (1:500, respectivamente), injetando-se, com uma seringa, $50 \mathrm{~mL}$ da solução dentro do prepúcio, sendo por fim, desinfetada a parte externa com o restante da solução.

O sêmen foi colhido pelo método da mão desenluvada (DIEHL et al.,1984). A coleta foi feita utilizando-se um manequim, sendo o ejaculado obtido em béquer graduado de $500 \mathrm{~mL}$, previamente revestido de gaze dupla medicinal, a fim de proteger os espermatozóides das radiações solares e das variações térmicas. A borda livre do béquer também foi coberta com gaze medicinal, dobrada cinco vezes, para reter a fração gelatinosa, a qual foi desprezada. O sêmen foi levado ao Laboratório de Reprodução Animal da FEI, sendo mantido em banho maria a $37^{\circ} \mathrm{C}$, até que as análises biológicas do sêmen fossem realizadas. Os outros materiais também foram mantidos a $37^{\circ} \mathrm{C}$, em banho maria ou sobre placa aquecedora. As avaliações mensais do sêmen consistiram de análises macro e microscópicas. Os parâmetros analisados no sêmen foram o volume, o pH, a cor, a motilidade progressiva (MP), o vigor (V), o grau de aglutinação (GA), a concentração espermática (CE), o índice de sobrevivência (IS), o índice de normalidade (IN) e o número de doses por ejaculado (NDE) (MIES FILHO, 1987). O volume foi determinado pela leitura direta no béquer, o $\mathrm{pH}$ por $\mathrm{pHmetro}$ digital, a cor foi determinada visualmente, sendo aceita como normal a cor branca leitosa. A MP foi 
avaliada colocando-se uma gota de sêmen diluído, na proporção de 1:5 (sêmen:citrato de sódio a $3 \%$, respectivamente) sobre uma lâmina (26 mm x $76 \mathrm{~mm}$ ) de microscópio coberta por lamínula $(22 \mathrm{~mm} \times 22 \mathrm{~mm})$, a qual foi levada ao microscópio óptico de contraste de fase, tendo sido estimada a porcentagem de motilidade individual dos espermatozóides de cada ejaculado, em que na escala de valores variou de 0 a 100\% (MIES FILHO, 1987). O V e o GA foram determinados juntamente com a MP. Para o V, foram atribuídos valores de 1 a 5 pontos, sendo os valores mais elevados indicadores de espermatozóides mais vigorosos e para o GA, valores de 0 a 5 pontos, em que os valores mais elevados indicaram sêmen mais aglutinado. A concentração espermática foi obtida utilizando-se câmara de Neubauerdupla, com sêmen diluído na proporção de 1:200 (sêmen:cloreto de sódio a 3\%, respectivamente), em pipeta de Thomas para contagem de glóbulos vermelhos. A contagem dos espermatozóides, na câmara de Neubauer, foi feita em microscópio de contraste de fase, com 40 aumentos, tendo sido contados cinco quadros de $0,04 \mathrm{~m}^{2}$ ou maiores (REED, 1969).
Após as avaliações, cada ejaculado foi dividido em três partes iguais, sendo que cada uma foi diluída com cada um dos três diluentes utillizados (Tabela 1) e novamente foram analisados a MP, o V e o GA referentes a cada diluente. Após a diluição do sêmen, o material foi colocado em frascos plásticos de $100 \mathrm{~mL}$, para ser conservado a $15^{\circ} \mathrm{C}$, tendo cada dose inseminante, em média, 5 bilhões de espermatozóides, com no mínimo $70 \%$ MP. Foram preparadas, no mínimo, três doses de cada diluente, sendo que duas foram utilizadas para IA e a terceira foi mantida para avaliações laboratoriais. As doses de sêmen foram agitadas, duas vezes ao dia, para que os espermatozóides que se encontravam no fundo dos frascos pudessem emergir e serem misturados ao diluente, dispersando-se os fatores tóxicos, resultantes do metabolismo dos mesmos.

As doses que se encontravam no laboratório foram, diariamente, analisadas até apresentarem 25\% de MP, seguindo as orientações de resultados previamente testados na laboratório de Reprodução Animal da UEM (MORAES e MOREIRA, 1995); para

Tabela 1 - Composição dos diluentes (BTS, Zorlesco-modificado e BTZOR) Table 1 - Diluents composition (BTS, Modified Zorlesco and BTZOR)

\begin{tabular}{|c|c|c|c|}
\hline $\begin{array}{l}\text { Produto } \\
\text { Product }\end{array}$ & $\begin{array}{l}\operatorname{BTS}^{1}(\mathrm{~g}) \\
\operatorname{BTS}^{1}(g)\end{array}$ & $\begin{array}{l}\text { Zorlesco modificado }{ }^{2}(\mathrm{~g}) \\
\quad \text { Modified Zorlesco }{ }^{2}(\mathrm{~g})\end{array}$ & $\begin{array}{l}\operatorname{BTZOR}^{3}(\mathrm{~g}) \\
\operatorname{BTZOR}^{3}(\mathrm{~g})\end{array}$ \\
\hline Glicose & 37,00 & 11,50 & 24,00 \\
\hline Glucose & & & \\
\hline Citrato de sódio & 6,00 & 11,65 & 4,80 \\
\hline Sodium citrate & & & \\
\hline Bicarbonato de sódio & 1,25 & 1,75 & 1,50 \\
\hline $\begin{array}{l}\text { Sodium bicarbonate } \\
\text { EDTA }^{4}\end{array}$ & 1,25 & 2,35 & 1,80 \\
\hline $\begin{array}{l}E D T A^{4} \\
\text { TRIS }^{5} \\
T R I S^{5}\end{array}$ & - & 6,50 & 3,50 \\
\hline $\begin{array}{l}\text { Cloreto de potássio } \\
\text { Potassium chloride }\end{array}$ & 0,75 & - & 0,40 \\
\hline $\begin{array}{l}\text { Penicilina G sódica } \\
\text { Penicilin } \mathrm{G}, \mathrm{Na}\end{array}$ & 1,00 & 1,00 & 1,00 \\
\hline $\begin{array}{l}\text { Diidrostreptomicina } \\
\text { Dihydrostretomycin }\end{array}$ & 1,00 & 1,00 & 1,00 \\
\hline $\begin{array}{l}\text { Ácido cítrico } \\
\text { Citric acid }\end{array}$ & - & 4,10 & 2,00 \\
\hline $\begin{array}{l}\text { Soro Fetal Bovino }(\mathrm{BSA}-\mathrm{V}) \\
\text { Bovine Serum Albumin }(B S A-V)\end{array}$ & - & 5,00 & 3,00 \\
\hline $\begin{array}{l}\text { Cisteína } \\
\text { Cysteine }\end{array}$ & - & 0,07 & 0,04 \\
\hline $\begin{array}{l}\text { Água deionizada (1) } \\
\text { Deionized water }(l)\end{array}$ & 1,00 & 1,00 & 1,00 \\
\hline
\end{tabular}

${ }^{1}$ BTS: Beltsville Thawing Solution (WOELDERS, 1992); ${ }^{2}$ ZORLESCO modificado (MORAES e MOREIRA, 1995), (Modified-Zorlesco [MORAES e MOREIRA, 1995]); ${ }^{3}$ BTZOR: Meio desenvolvido no laboratório da UEM (MORAES e MOREIRA, 1995) (BTZOR: media developed at Universidade Estadual de Maringá lab); ${ }^{4}$ EDTA: Etilenodiamino tetracetato (EDTA: ethylenediamine tetracetate); ${ }^{5}$ TRIS: Hidroxi-metil-amino-metano (TRIS: Hydroxymethyl aminomethane). 
cada dia de conservação de cada dose foram preparadas duas lâminas com esfregaços, as quais, após secas, foram coradas (WILLIAMS, 1920, modificada por LAGERLÖFF, 1934), em que se contaram, no mínimo, 200 células entre as duas lâminas, em microscópio de contraste de fase de $1000 \mathrm{X}$, para avaliar o percentual de células normais e as diversas anormalidades referentes a cada dia de vida útil de cada sêmen (MIES FILHO, 1987; PIG INTERNATIONAL, 1994). As anormalidades foram classificadas em graves ou maiores e leves ou menores, de acordo com MIES FILHO (1987) e PIG INTERNATIONAL (1994). Foram considerados como defeitos graves ou maiores o subdesenvolvimento, formas duplas, "knobbed sperm, decapited sperm, pouch formation", cabeça piriforme, cabeça estreita na base, cabeça de contorno anormal, cabeças pequenas, cabeça solta, "corkscrew defect", defeitos de peça intermediária, gotas proximais e cauda enrolada. Como defeitos leves ou menores, foram consideradas cabeças estreitas, cabeça pequena normal, cabeça gigante, cabeças soltas normais, acrossomas destacados, implantação abaxial, gotas citoplasmáticas distais, cauda dobrada e cauda enrolada na porção terminal.

Nas avaliações estatísticas deste experimento, foram incluídos a MP, o V, o GA, as porcentagens de células normais e as porcentagens de células anormais diárias e totais/ano e o período de conservação de sêmen para cada diluente.

Os dados foram submetidos às análises de variância e regressão, utilizando-se o seguinte modelo:

$$
Y_{i j k l}=\mu+D_{i}+M_{j}+C_{k}+e_{i j k l}
$$

em que $Y_{\mathrm{ijkl}}$ é observação "l" referente ao sêmen do cachaço "k", no mês " $j$ " submetido ao tratamento " $\mathrm{i}$ "; $\mu$, constante geral; $\mathrm{D}_{\mathrm{i}}$, efeito do diluente $\mathrm{i}, \mathrm{i}=1$; $2 ; 3 ; \mathrm{M}_{\mathrm{j}}$, efeito do mês $\mathrm{j}, \mathrm{j}=1 ; \ldots ; 12 ; \mathrm{C}_{\mathrm{k}}$, efeito do cachaço $\mathrm{k}, \mathrm{k}=1 ; 2 ; 3 ; \mathrm{e}_{\mathrm{ijkl}}$, erro aleatório associado a cada observação $\mathrm{Y}_{\mathrm{ijkl}}$.

As diferenças entre os efeitos dos diluentes foram avaliadas pelo teste de Newman-Keul's e análise de regressão, utilizando-se identidade de modelos, a $5 \%$.

\section{Resultados e Discussão}

Os resultados referentes ao período de conservação do sêmen até $25 \%$ de MP, a porcentagem de motilidade progressiva inicial (MPi), vigor inicial (Vi), grau de aglutinação inicial (GAi), a porcentagem de normalidade inicial, a porcentagem de anormalidade inicial (Ai) e a porcentagem de anormalidades totais (At/ano) são apresentados na Tabela 2. Não foram observadas influências iniciais dos diluentes sobre a $\mathrm{MPi}$, o Vi, o GAi e as porcentagens de normalidade e anormalidade espermáticas (Tabela 2).

De acordo com o teste de média de Newman Keul's, o BTZOR $(4,94 \pm 0,30$ dias $)$ foi o meio que conservou o sêmen por mais tempo $(\mathrm{P}<0,05)$, seguido pelo ZOR (4,62 $\pm 0,29$ dias) e BTS $(3,27 \pm 0,30$ dias $)$, tendo havido diferença $(\mathrm{P}<0,05)$ também entre estes dois últimos.

As análises de regressões mostraram que, durante o decorrer do ano, o Vi $\left(\mathrm{R}^{2}=0,29\right)$ e o GAi $\left(\mathrm{R}^{2}=0,30\right)$ apresentaram diferenças $(\mathrm{P}<0,01)$ em relação aos meses do ano, sendo que em janeiro foi observada a melhor média de $\mathrm{V}(2,96 \pm 0,39$ pontos $)$ e a pior média $(2,06 \pm 0,51$ pontos $)$ no mês de junho. O GA também foi influenciado pela época do ano $(\mathrm{P}<0,05)$, em que a melhor média foi observada em março $(1,44 \pm 0,19$

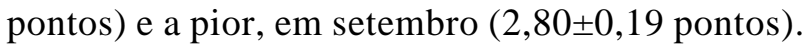

A melhor $(\mathrm{P}<0,05)$ média de MP foi de $53,21 \% \pm 9,02$ em abril, na época do calor, e a pior $(\mathrm{P}<0,05)$ média foi de 30,37\% $\pm 13,18$ em junho, na época do frio.

Aplicando-se a análise de identidade de modelos, observou-se que a MP apresentou comportamento quadrático $(\mathrm{P}<0,01)$ com o decorrer do tempo de conservação do sêmen, quando foram usados os diluentes BTS e ZOR, enquanto, ao se usar o diluente BTZOR, este comportamento foi cúbico $(\mathrm{P}<0,01)$, sendo que o coeficiente de determinação geral foi de 0,58. A MP média apresentada nos três diluentes durante o período de armazenamento foi de 40,72; 50,91 e 40,76\% para o BTS, o ZOR e o BTZOR, respectivamente.

$\mathrm{O}$ V apresentou comportamento linear $(\mathrm{P}<0,05)$ em relação aos diluentes BTS e ZOR e quadrático $(\mathrm{P}<0,05)$ para o BTZOR, cujo coeficiente de determinação geral foi de 0,31 . O V médio observado nos três diluentes, durante o armazenamento do sêmen, foi de 2,54; 2,77 e 2,50 pontos para o BTS, o ZOR e o BTZOR, respectivamente. Os resultados mostraram que o comportamento da MP e do $\mathrm{V}$ foi superior no diluente BTZOR em relação ao BTS e ao ZOR, porém o GA teve comportamento linear para todos os diluentes $(\mathrm{P}<0,01)$, sendo os valores médios de 2,38 pontos no BTS, 1,79 pontos no ZOR e 1,94 pontos no BTZOR, cujo coeficiente de determinação geral foi de 0,37 . O sêmen diluído em todos os meios diluentes apresentou decréscimo progressivo de qualidade (Figuras 1, 2 e 3). 
No caso do BTZOR, cuja MP apresentou comportamento cúbico, ou no caso do $\mathrm{V}$, em que o comportamento foi quadrático, não foi encontrada explicação biológica, pois esperar-se-ai que este meio tivesse comportamento idêntico aos meios BTS e ZOR.

As porcentagens de células espermáticas normais avaliadas durante o decorrer dos dias de conservação do sêmen, em relação aos diluentes e aos meses do ano, não foram afetadas por nenhuma dessas variáveis. As porcentagens de espermatozóides morfologicamente anormais de todos os ejaculados não foram afetados pelos diluentes (Tabela 2).

Constatou-se que houve diferenças $(\mathrm{P}<0,05)$ quanto aos dias de conservação do sêmen em condições de serem usados na IA (25\% MP), tendo o BTS apresentado a média de conservação de $3,27 \pm 0,30$ dias; o ZOR, de 4,62 $\pm 0,29$ dias; e o BTZOR, de 4,94 $\pm 0,30$ dias, mostrando que o BTZOR conservou o sêmen com espermatozóides viáveis à IA por maior período que o ZOR e o BTS. Notou-se, por estes resultados, que o BTZOR e o ZOR foram superiores ao BTS, quanto ao período de conservação do sêmen, o que está de acordo com as observações de
WABERSKI et al. (1994), ao analisarem zolesco e BTS. Isto talvez se deva à presença da albumina sérica de bovino, substância que tem propriedades anti-tóxicas, diminuindo os efeitos dos catabólitos resultantes do metabolismo dos espermatozóides, além de apresentar propriedades anti-aglutinantes.

Entre as pesquisas sobre os dias de conservação do sêmen, considerando ou não a MP, destacam-se MORAES e MURATA (1992), que encontraram MP de 63,0 e 70,1\% para sêmen de reprodutores Duroc e Landrace, diluídos em BTS, respectivamente, ou de 77,3 e 79,3\%, quando o sêmen desses mesmos reprodutores foi diluído em Zorlesco original, respectivamente. MORAES e MOREIRA (1995), trabalhando com diferentes diluentes, obtiveram, em média, 5,3 dias de conservação do sêmen em condições de ser utilizado em IA e $29,1 \%$ de MP, quando o sêmen foi diluído em BTS; 6,7 dias de conservação e 36,1\% de MP, quando o sêmen foi diluído em Zorlescomodificado; e 7,8 dias de conservação e $39,8 \%$ de MP, ao ser diluído em BTZOR, resultados que são em geral, superiores aos obtidos neste estudo. Já BRUNEL e GOTTARDI (1982), utilizando como

Tabela 2 - Médias e erros-padrão de motilidade progressiva inicial (MPi),.vigor inicial (Vi), grau de aglutinação inicial (GAi), índice de normalidade inicial (Ni), índice de anormalidade inicial (Ai), índice de anormalidades totais/ano (At/ano) e do período máximo de conservação dos espermatozóides viáveis à inseminação artificial, de acordo com o diluente usado

Table 2 - Means and standard errors of initial progressive motility (IPM), initial vigor (IV), initial agglutination degree (IAG), initial normality index (IN), initial abnormality index (IA), total abnormalities/year index (TA/year) and the longest conservation period of spermatozoa viable for artificial insemination, according to used diluents

\begin{tabular}{|c|c|c|c|}
\hline \multirow[b]{2}{*}{$\begin{array}{l}\text { Parâmetro } \\
\text { Parameter }\end{array}$} & \multicolumn{3}{|c|}{$\begin{array}{l}\text { Diluente } \\
\text { Diluents }\end{array}$} \\
\hline & $\begin{array}{l}\mathrm{BTS}^{1} \\
\text { BTS }^{1}\end{array}$ & $\begin{array}{l}\text { Zorlesco modificado } \\
\text { Modified Zorlesco }^{2}\end{array}$ & $\begin{array}{l}\mathrm{BTZOR}^{3} \\
\text { BTZOR }^{3}\end{array}$ \\
\hline $\begin{array}{l}\text { Mpi (\%) } \\
I P M(\%)\end{array}$ & $70,83 \pm 3,17^{\mathrm{a}}$ & $79,40 \pm 3,17^{\mathrm{a}}$ & $77,43 \pm 3,17^{a}$ \\
\hline $\begin{array}{l}\mathrm{Vi}(\text { pontos }) \\
I V(\text { scores })\end{array}$ & $3,0 \pm 0,12^{\mathrm{a}}$ & $3,3 \pm 0,12^{\mathrm{a}}$ & $3,4 \pm 0,12^{\mathrm{a}}$ \\
\hline $\begin{array}{l}\text { GAi (pontos) } \\
\text { IAD (scores) }\end{array}$ & $1,6 \pm 0,19^{a}$ & $1,0 \pm 0,19^{\mathrm{a}}$ & $1,1 \pm 0,19^{\mathrm{a}}$ \\
\hline $\begin{array}{l}\mathrm{Ni}(\%) \\
I N(\%)\end{array}$ & $85,82 \pm 1,54^{\mathrm{a}}$ & $85,75 \pm 1,54^{\mathrm{a}}$ & $87,35 \pm 1,54^{a}$ \\
\hline $\begin{array}{l}\mathrm{Ai}(\%) \\
\mathrm{IA}(\%)\end{array}$ & $14,18 \pm 1,54^{\mathrm{a}}$ & $14,23 \pm 1,54^{\mathrm{a}}$ & $12,65 \pm 1,54^{\mathrm{a}}$ \\
\hline $\begin{array}{l}\text { At/ano }(\%) \\
\text { TA/year }(\%)\end{array}$ & $12,50 \pm 1,32^{\mathrm{a}}$ & $14,54 \pm 1,32^{\mathrm{a}}$ & $15,30 \pm 1,32^{\mathrm{a}}$ \\
\hline $\begin{array}{l}\text { Dias de conservação } \\
\text { Conservation days }\end{array}$ & $3,27 \pm 0,30^{\mathrm{a}}$ & $4,62 \pm 0,29^{b}$ & $4,94 \pm 0,30^{c}$ \\
\hline
\end{tabular}

1 Beltsville Thawing Solution, ${ }^{2}$ Zorlesco-modificado (MORAES e MOREIRA, 1995) (Modified-Zorlesco (MORAES e MOREIRA, 1995); ${ }^{3}$ Meio desenvolvido no laboratório da Universidade Estadual de Maringá (UEM) (MORAES e MOREIRA, 1995) (Media developed at Universidade Estadual de Maringá lab).

Médias com sobrescritos diferentes, nas linhas, diferem $(P<.05)$.

Means, within a row, with different superscripts are different $(P<.05)$. 


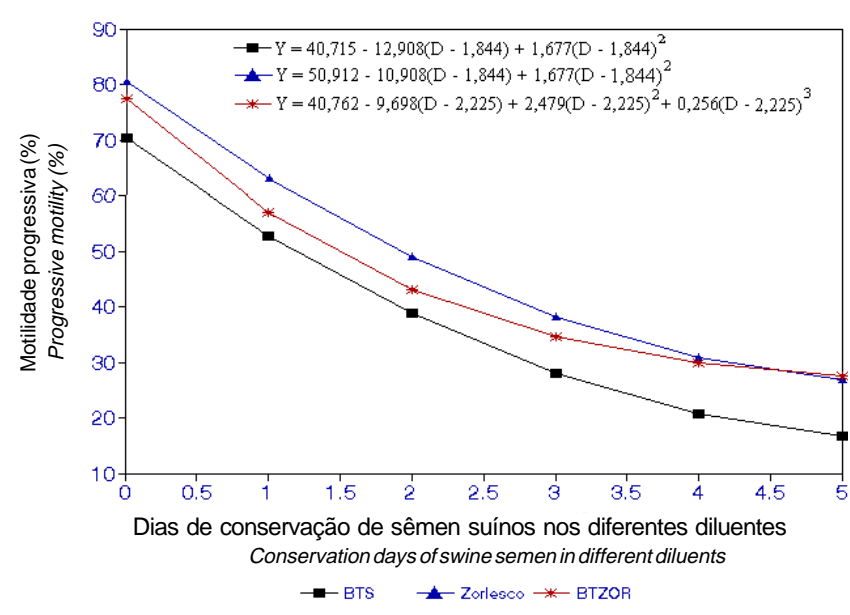

Figura 1 - Comportamento da motilidade do sêmen suíno durante os dias de conservação nos diluentes Beltsville Thawing Solution, Zorlesco-modificado e BTZOR (meio desenvolvido na UEM).

Figure 1 - Motility behavior of swine semen during the conservation days in Beltsville Thawing Solution, Modified Zorlesco and BTZOR (developed media at UEM) diluents.

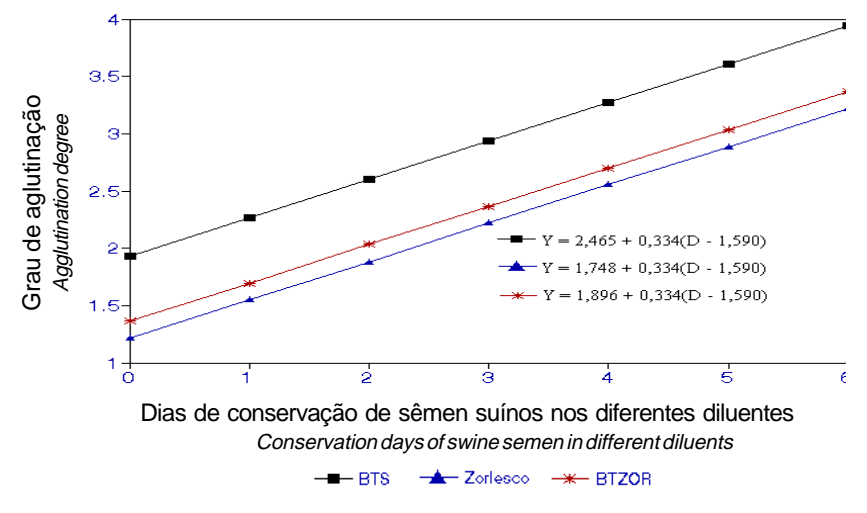

Figura 3 - Comportamento do grau de aglutinação do sêmen suíno durante os dias de conservação nos diluentes Beltsville Thawing Solution, Zorlesco-modificado e BTZOR (meio desenvolvido na UEM).

Figure 3 - Agglutination degree behavior of swine semen during the conservation days in BTS, Modified Zorlesco and BTZOR (developed media at UEM) diluents.

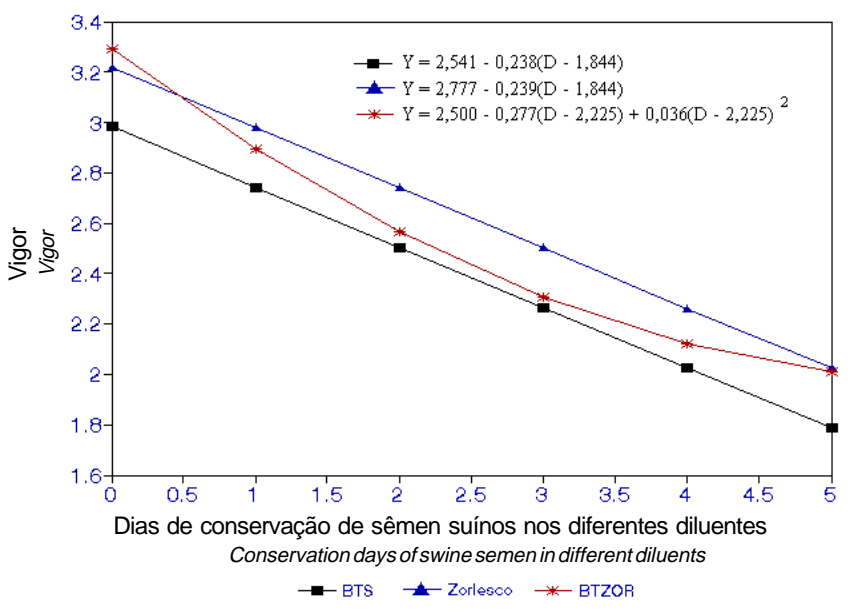

Figura 2 - Comportamento do vigor do sêmen suíno durante os dias de conservação nos diluentes Beltsville Thawing Solution, Zorlesco-modificado e BTZOR (meio desenvolvido na UEM).

Figure 2 - Vigor behavior of swine semen during the conservation days in BTS, Modified Zorlesco and BTZOR (developed media at UEM) diluents.

diluente de sêmen de suíno o Zorlesco original, verificaram que, depois de $2,4,5,8,10$ e 12 dias de conservação, as motilidades progressivas foram de 65,$41 ; 60,41 ; 56,60 ; 53,91 ; 50,12$ e $48,16 \%$, respectivamente. Além disto, destacaram que a motilidade mínima necessária para bons resultados na IA, com sêmen resfriado, foi de $50 \%$, quando neste experimento se considerou $25 \%$.

AALBERS et al. (1983) conduziram dois experimentos de campo, em que analisaram ejaculados de cachaço, os quais foram diluídos em BTS e Zorlesco e conservados em $18 \pm 2^{\circ} \mathrm{C}$. Efetuaram a IA no terceiro dia de conservação do sêmen e verificaram que as MP foram de 42 e $56 \%$, respectivamente, estando estes resultados próximos aos dados encontrados neste experimento (Figura 1). GALLI e BOSISIO (1988) utilizaram 22 ejaculados de suínos conservados entre 15 e $16^{\circ} \mathrm{C}$, diluídos em meio Zorlesco modificado, e verificaram que, um dia após a colheita e a diluição dos ejaculados, a porcentagem média de MP foi de 60,87\%. REVELL e GLOSSOP (1989), testando os 
diluentes Zorpva e Reading, combinados com antibióticos, verificaram que as MP, nos dias 1, 4, 5 e 6 , foram de 50, 50, 30 e 5\% para o Zorpva e de 60, 55, 55 e $40 \%$ para o Reading, respectivamente, dados estes que se assemelham ao deste experimento (Figura 1), embora os meios sejam diferentes.

SCHEID (1991) testou os diluentes BTS, Reading e Kiev, quanto à motilidade espermática do sêmen preservado por seis dias, e verificou que houve variação de 73,3 (dia 1) a 64,6\% (dia 6) para sêmen diluído em BTS, valor que pode ser considerado elevado, diante dos resultados obtidos aqui (Figura 1), com o BTS.

MURATA e MORAES (1992) trabalharam com os diluentes Kiev, BTS e Zorlesco, quando observaram variação de 78,29 (primeiro dia) a 38,93\% (sexto dia) no ejaculado diluído em Zorlesco, 64,43 (primeiro dia) a $22,84 \%$ (sexto dia) no sêmen diluído em Kiev e 66,81 (primeiro dia) a 36,93\% (sexto dia), quando o sêmen foi diluído em BTS, sendo os melhores resultados para os diluentes Zorlesco e BTS. Nesta pesquisa, observou-se, no entanto, que o BTZOR foi melhor que o Zorlesco e o BTS, inferior. ALEXOPOULOS et al. (1996) também verificaram reduções significtivas da MP de sêmen conservado em BTS, a partir de 48 horas.

Em relação ao $\mathrm{V}$, foram observados comportamentos de regressão linear (BTS e Zorlesco-modificado) e quadrático (BTZOR) e em relação ao GA, verificou-se comportamento linear em todos os diluentes. Observou-se que o $\mathrm{V}$ diminuiu e o GA, aumentou, tendo estes comportamentos ocorrido de modo progressivo com o passar dos dias, ao serem analisados por regressão, via identidade de modelos. Estes resultados estão de acordo com as observações feitas por MORAES e MOREIRA (1995), num trabalho com BTS, Zorlesco-modificado e BTZOR. Também MORAES e MURATA (1992) obtiveram resultados semelhantes quanto ao GA, mas trabalharam com sêmen conservado e diluído em BTS, Zorlesco e Kiev, em que os valores variaram de 1,5 pontos no primeiro dia a 2,52 pontos no sexto dia, para o BTS; 0,84 pontos no primeiro dia a 1,42 pontos no sexto dia para o Zorlesco; e 1,30 pontos no primeiro dia a 3,06 pontos no sexto dia para o Kiev.

Foi constatado efeito $(\mathrm{P}<0,01)$ de regressão quadrática quanto aos meses do ano sobre o $\mathrm{V}$ e o $\mathrm{GA}$, sendo que os melhores valores ocorreram em janeiro e março e os piores, em junho e setembro, respectivamente. Estes resultados são contraditórios, pois esperar-se-iam melhores resultados de qualidade de sêmen no inverno em vez do verão, visto que REYES (1995) mostrou que a alta temperatura causou flutuação dramática na MP de ejaculados de mais de $45 \%$ dos reprodutores das Filipinas, destinados à IA.

GALLI e BOSISIO (1988), ao analisarem 22 ejaculados diluídos em Zorlesco, verificaram que o índice de patologias de um dia depois da diluição foi de $21,70 \%$, o que está acima dos 14,54\% (4,62 dias de conservação) obtidos neste experimento (Tabela 2).

Não houve efeito dos diferentes diluentes sobre as anormalidades espermáticas, durante a conservação do sêmen, tendo os valores se situado dentro de faixas tecnicamente aceitáveis, com anormalidades abaixo de $30 \%$, indicando que o sêmen, sob este ponto de vista, teria capacidade fecundante. XU et al. (1996), ao conservarem sêmen diluído em BL-1, meio semelhante ao BTS, mantido a $17^{\circ} \mathrm{C}$ por sete dias, obtiveram $95 \%$ de espermatozóides normais com todos os ejaculados, valor um pouco superior aos observados nesta pesquisa. Vale destacar que os resultados de PEDERSEN et al. (1995) mostraram que as patologias elevadas reduzem a capacidade fecundante dos espermatozóides. BERGER et al. (1996) também mencionaram a relação entre a fecundidade dos espermatozóides e a normalidade morfológica, embora citem as dificuldades de incluir tais análises em uma rotina.

\section{Conclusões}

O BTZOR apresentou o maior período de conservação de sêmen, seguido do Zorlesco-modificado e do BTS. Considerando a motilidade progressiva, o vigor e o grau de aglutinação analisados por identidade de modelos, todos os diluentes apresentaram-se viáveis à inseminação artificial de suínos.

\section{Referências Bibliográficas}

AALBERS, J.G., RADEMAKER, J.H.M., GROOTEN, H.J.G. et al. 1983. Fecundity of boar semen stored in BTS, Kiev, Zorlesco and Modena extenders under field conditions. J. Anim. Sci., 57(suppl.1):314-315.

ALEXOPOULOS, C., BOSCOS, C., SARATSIS, C. et al. 1996. The effect of storage time and number of spermatozoa per insemination dose on semen characteristics and fertilizing capacity of boar semen diluted with Beltsville Thaw Solution (BTS) extender. Anim. Sci., 62(3):599-604.

BARITEAU, F., BUSSIERE, J., COUROT, M. et al. 1977. Insemination artificielle porcine ameliorations techniques, resultats recents. J. Rech. Porc. en France, 1:11-14.

BERGER, T., ANDERSON, D.L., PENEDO, M.C.T. 1996. Porcine sperm fertilizing potencial in relationship to sperm functional capacities. Anim. Reprod. Sci., 44(4):231-239. 
BRUNEL, L., GOTTARDI, L. 1982. Nuovi mestrui per una lunga conservazione de nemospermi di verro. Sel. Vet., 23(4/5):417-427.

DIEHL, J.R., DAY, B.N., STERVERMER, E.J. 1984. Artificial insemination in swine. Porc. Ind. Hand., 10(4/5):417-427.

GALLI, A., BOSISIO, M. 1988. Quality of semen stored at 15/ $16^{\circ} \mathrm{C}$ as related to fertility of artificially inseminated swine. Theriogenology, 30(6):1185-1189.

JOHNSON, L.A., AALBERS, J.G., WILLEMS, C.M.T. et al. 1982. Use of boar spermatozoa for artificial insemination. III. Fecundity of boar spermatozoa stored in Beltsville Liquid and Kiev Extenders for three days at 18. J. Anim. Sci., 54(1):132-141.

JOHNSON, L.A., AALBERS, J.G., GROOTEN, H.J.G. 1988. Artificial insemination of swine: fecundity of boar semen stored in Beltsville TS (BTS), modified modena (MM), or MR-A and inseminated on one, three and four days after collection. Zuchthygiene, 23(2):49-55.

LAGERLÖFF, N. 1934. Morphogischi untersuchungen uber veranderugen in espermbid in den hoden bei bullen mituerminderter oder aufgehobener ferdilitat. Acta pathol. and Microbiol. Scand, 19(suppl.):sp.

MIES FILHO, A. 1987 Inseminação artificial. 6.ed., Porto Alegre: Editora Sulina. v.2. 783p.

MORAES, G.V., MURATA, L.S. Efeito dos diferentes diluidores sobre o grau de aglutinação do sêmen de suíno resfriado. In: REUNIÃO ANUAL DA SOCIEDADE BRASILEIRA DE ZOOTECNIA, 29, 1992, Lavras, Minas Gerais. Anais... Lavras: SBZ, 1992. p.234.

MORAES, G.V., MOREIRA, I. Diluentes na conservação de sêmen resfriado de suíno. In: REUNIÃO ANUAL DA SOCIEDADE BRASILEIRA DEZOOTECNIA, 32, 1995, Brasília, DF. Anais... Brasília: SBZ, 1995. p.140.

MURATA, L.S., MORAES, G.V. Aspectos econômicos dos diluidores Kiev (SK), Beltsville Thawing Solution-BTS (SB) e Zorlesco (SZ) na inseminação artificial de suínos. In: REUNIÃO ANUAL DA SOCIEDADE BRASILEIRA DE ZOOTECNIA, 29, 1992, Lavras, Minas Gerais. Anais... Lavras: SBZ, 1992. p.236.

PAQUIGNON, M., BUSSIERE, J., BARITEAU, F. 1987. Resultats recents en matiere de technologie de la conservation de la semence de verrat. J. Rech. Porc. en France, 19:63-78.

PEDERSEN, N.P. THE INTERNATIONAL COMMITTEE FOR PIG BREEDING, HEALTH AND PRODUCTION et al. Handling semen. In: CONFERÊNCIA INTERNACIONAL SOBRE CIÊNCIA E TECNOLOGIA DE PRODUÇÃO E INDUSTRIALIZAÇÃO DE SUÍNOS,1, 1995, Campinas. Anais... Campinas, 1995. p.65-69.
PIG INTERNATIONAL. 1994. Testing semen for quality. Pig Int., 24(5):15-16.

PIG INTERNATIONAL. 1996. Looking at sperm. Pig Int., 26(1):11-12.

REED, H.C.B. 1969. Artificial insemination and fertility of the boar. Br. Vet. J., 125(6):272-280.

REVELL, S.G., GLOSSOP, C.E.A. 1989. Long-time ambient temperature diluent for boar semen. Anim. Prod., 48:579-584.

REYES, I.M. 1995. Air conditioning raises boar semen standarts. Pig Int., 25(3):16-18.

SCHEID, I.R. 1991. Inseminação artificial em suínos: diluentes para a conservação do sêmen no estado líquido. Concórdia: EMBRAPA/CNPSA. 5p. (Comunicado técnico, n.12).

SILVEIRA, P.R.S. 1983. Efeito do diluente Kiev sobre a fertilidade de porcas inseminadas artificialmente. Santa Maria: UFSM, 9 p. (Seminário).

WABERSKI, D., WEITZE, K.F., LIETMANN, C. et al. The initial fertilizing capacity of longterm-stored liquid boar semen following pre-and postovulatory insemination. Theriogenology, 41(7):1367-1377.

WOELDERS, H. 1992. Maintaining quality of boar sperm during storage and transportation. PIGS - Misset, 8:22-23.

XU, X., SETH, P.D., HARBISON, P.S. et al. 1996. Semen dilution for assessment of boar ejaculate quality in pig IVM and IVF systems. Theriogenology, 46(8):1325-1337.

ZAVOS, P.M., CORREA, J.R., ZARMSKOUPIS-ZAVOS, P.N. 1996. Measurement of the sperm motility index via the sperm quality analyser and its relationship to qualitative sperm parameters. Theriogenology, 46(3):421-427.

Recebido em: 18/10/99

Aceito em: 04/10/00 